\title{
Graph Theoretical Analysis of Interictal EEG Data in Epilepsy Patients during Epileptiform Discharge and Non-discharge
}

\author{
Steven M.A. CARPELS, Yusuke YAMAMOTO and Yuko MIZUNO-MATSUMOTO \\ University of Hyogo, Graduate school of Applied Informatics, 7-1-28 Minatojima-minamimachi, \\ Chuo-ku, Kobe, Hyogo 650-0047, Japan
}

\begin{abstract}
Graph theoretical analysis has recently been used to study brain function. This study aims to compare the functional brain networks derived from electroencephalography (EEG) of 10 patients suffering from epilepsy with 10 healthy subjects based on graph theory. Five epochs per healthy subject, and ten epochs (during epileptiform discharge and non-discharge) per patient were selected and analyzed using wavelet-crosscorrelation analysis. The clustering coefficient, characteristic path length, small-worldness, and nodal betweenness centrality were calculated using graph analysis. The results showed that in the patients, Wavelet-crosscorrelation Coefficients (WCC) were significantly higher, and clustering and path length were significantly lower during discharge compared with the healthy subjects, along with alterations in the hub regions. These results suggest a loss of small-world topology in the functional brain network of epilepsy patients. A loss of small-world topology was found even during non-discharge, therefore network indices might aid to distinguish epilepsy patients from healthy individuals.
\end{abstract}

Keywords: Electroencephalography, Epilepsy, Graph theory

\section{INTRODUCTION}

Epilepsy is one of the most common neurological disorders, affecting $1 \%$ of the world's population, and is characterized by epileptic seizures originating from paroxysmal synchronous neuronal firing [1,2]. Several studies have reported that electroencephalography (EEG) could be an important tool for the diagnosis of epilepsy and to study epilepsy processes [2-5]. The EEG of epilepsy patients during non-epileptic seizures is characterized by the appearance of epileptiform discharges and is referred to as interictal EEG $[4,5]$. While the detection of interictal epileptiform discharges in EEG is important for diagnosis, it has been reported that around $10 \%$ of epileptic patients never show discharges [2,3]. These studies suggest that diagnosing epilepsy with high sensitivity is still difficult. It has been reported that synchronization between EEG time series from different brain regions differs between epilepsy patients and healthy subjects in the interictal state [2]. This study concluded that analyzing functional connectivity may help in the diagnosis of epilepsy.

Functional connectivity in the brain is defined as the statistical interdependencies between signals of brain activity recorded from distant brain regions by neuroimaging techniques $[6,7]$. These interdependencies between time series are considered to reflect the functional interactions between different brain areas, and can be derived from various analysis methods [8]. Characterization and quantification of the functional connectivity in the human brain may help reveal changes in neuronal activity and connection patterns between brain areas, and allow us to understand how these changes give rise to disturbances in brain function [9]. Mizuno-Matsumoto et al. [10] reported that a method called wavelet-crosscorrelation analysis of MEG data could help visualize the initiation and propagation of epileptiform activity in the brain of epilepsy patients. Previous work suggested that focal lesions caused by brain tumors disturb not only the localized brain function, but also the widespread functional connectivity between brain areas [11]. Brain disorders may not only be related to a dysfunction of a certain brain area, but also to the way brain areas are functionally connected [12]. Brain function seems to be dependent on the spatial distribution (the topology) of the functional connections.

In modern neuroscience, a new method using graph theory has been proposed to characterize and quantify the topology of the brain as a network. The brain can be considered a complex anatomical or functional network of neurons, neuron populations, or brain regions [8, 13, 14]. A graph is an abstract mathematical representation of a real-life network. In graph theory, the nodes of a network are referred to as vertices, and the connections (links) between these nodes as edges [15]. Watts and Strogatz have discovered that other than regularly connected networks and completely randomly connected networks, there is a class of networks called "small-world" networks [16]. Small-world networks have many local connections (high 
clustering) between nodes, and a few random longdistance connections (short path lengths) between nodes. A small-world network is considered to be an optimal network, and many types of real-life networks have been shown to have small-world properties $[15,17]$. A lot of research has revealed small-world topology in anatomical and functional networks of the brain in animals and in humans. Studies based on MRI found a small-world configuration in both the anatomical network [13] and the functional network [18] of the human brain. Previous work based on EEG revealed small-world properties in the brain functional networks of healthy individuals. These studies revealed a loss of optimal brain function due to a loss of small-world topology in the brain network of Schizophrenia [19] and Alzheimer [7] patients, and suggested that network measures may aid in the diagnosis of neuropsychiatric diseases. Studies based on fMRI found altered small-world topology in epilepsy patients compared to healthy adults [20,21]. Another study based on intracranial electrocorticographic recordings in epilepsy patients found different network topologies during the ictal, preictal, and postictal periods [22].

The International Journal of Affective engineering is a scientific journal that fuses a wide range of academic fields, such as philosophy, education, psychology, art, politics, economics, social sciences, as well as medical science, physiology, chemistry, machinery, and information science [23]. Affective engineering is a science that utilizes sensitivity to contribute to society, but is not limited by the conventional framework of social sciences and natural sciences. The processing of information in the brain is important for sensitivity and responsiveness to external stimuli. Our study aimed to approach sensitivity from a medical perspective.

Using wavelet-crosscorrelation analysis and graph theoretical analysis of scalp recorded EEG, this study aimed to examine whether or not the functional connectivity and the small-world properties of the brain network of healthy individuals differ from epilepsy patients. While previous studies used fMRI recordings and graph theory to examine the network topology of healthy adults and epilepsy patients $[20,21]$, in this paper we focus on scalp EEG recordings, which have a high temporal resolution suitable for analyzing rapidly changing brain signals. The second purpose of this study was to examine the interictal period of the EEG of the epilepsy patients, and compare the functional connectivity and the small-worldness of the brain network between during epileptiform discharge and during non- discharge. A study based on MRI found that not only the small-world topology, but also the centrality of the brain network of patients with Alzheimer's disease is different from healthy individuals [24]. Following this study, we lastly aimed to examine whether or not the nodal betweenness centrality of the brain network differs between healthy subjects and epilepsy patients, and between during discharge and during non-discharge in the interictal period.

\section{METHODS}

\subsection{Recording and selecting of the data}

EEG data was obtained by scalp recordings from 10 healthy subjects ( 5 males and 5 females; mean age $=24.40$ \pm 2.46 years) and 10 individuals who were diagnosed with epilepsy ( 4 males and 6 females; mean age $=41.30 \pm 14.44$ years). The healthy individuals were adults whose EEG showed no abnormalities. There is an age difference between the healthy subjects and patients, but based on previous studies, the functional network of the brain shows differences between children and healthy adults [25], and between healthy adults and elderly individuals [26]. Another study based on EEG divided healthy adults in 3 age groups, and showed a different brain functional network only between young adults (ages 16-45), middle-aged adults (ages 50-70), and elderly people (age >70) [27]. In our study, the possibility that the age difference could affect the results to some degree cannot be excluded, however based on these studies, this possible influence might be minimal.

All 10 epilepsy patients suffered from generalized epilepsy. No epileptic focus was present in any of the patients' brains. Table 1 shows the medical diagnosis of each patient. Epileptiform discharges could be observed in each patient's EEG. The EEGs of all subjects were measured during resting-state with eyes closed, and there was no sound at the time of recording. To record the EEG data, scalp electrodes (19 channels) were placed according to the International 10-20 electrode positions system (Figure 1). The sampling frequency was $500 \mathrm{~Hz}$.

Table 1: Medical diagnosis of the epilepsy patients

\begin{tabular}{|c|c|c|c|}
\hline $\begin{array}{c}\text { Patient } \\
\text { number }\end{array}$ & $\begin{array}{c}\text { Age } \\
\text { [years] }\end{array}$ & Gender & Type of epilepsy \\
\hline 1 & 32 & Male & Autonomic seizure \\
\hline 2 & 34 & Male & Autonomic seizure \\
\hline 3 & 52 & Female & Autonomic seizure \\
\hline 4 & 48 & Female & Grand mal \\
\hline 5 & 25 & Female & Grand mal \\
\hline 6 & 33 & Female & Autonomic seizure \\
\hline 7 & 56 & Female & Grand mal \\
\hline 8 & 61 & Female & Grand mal \\
\hline 9 & 23 & Male & Autonomic seizure \\
\hline 10 & 59 & Male & Grand mal \\
\hline
\end{tabular}




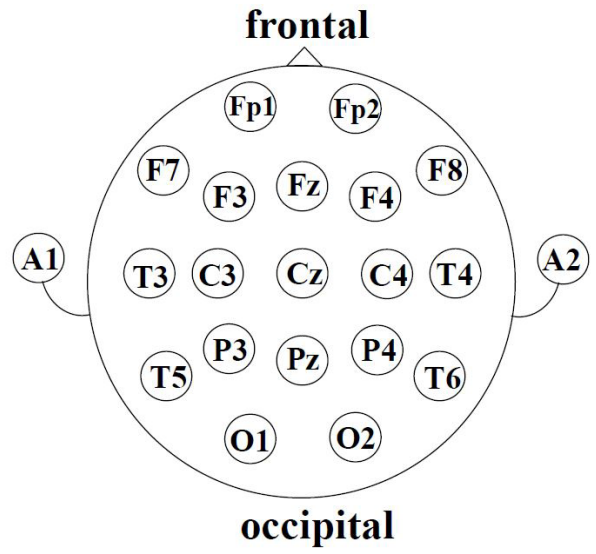

Figure 1: The international 10-20 electrode positions system

A bandpass filter was set between $0.5 \mathrm{~Hz}$ and $30.0 \mathrm{~Hz}$. For each healthy subject's measured EEG, five artifactfree epochs of 2 seconds were carefully selected for analysis by visual inspection (in total 50 epochs). For each epilepsy patient's measured EEG, five artifact-free 2-second-epochs that had epileptiform discharge (in total 50 epochs), and five artifact-free 2-second-periods that showed no discharge (in total 50 epochs) were selected and carefully distinguished by visual inspection. Within each patient's interictal EEG, the epileptiform discharge epochs and non-discharge epochs were selected with a time difference of 1 to 3 minutes from each other on the time course of the EEG. In this paper, we did not investigate the time course of the EEG, but following previous studies, we distinguished and selected several discharge and non-discharge epochs repeatedly per subject [2,28]. The selected epochs were then averaged in order to achieve reliable statistical comparison of network measures between during discharge and during non-discharge, as well as between healthy subjects and patients. This research was approved by the Ethics Committee of the Graduate School of Applied Informatics of the University of Hyogo (UHGSAI-2018-9). All subjects agreed to be measured and gave written informed consent.

\subsection{Analysis methods}

We analyzed all selected EEG epochs of the healthy subjects and the epilepsy patients based on a method called wavelet-crosscorrelation analysis in the alpha 1 band $(8.07-10.87 \mathrm{~Hz})$ to obtain correlations between time series from different brain areas. The correlation strengths were visualized in graphs to reveal functional connectivity patterns. High reliability cannot be obtained by analyzing low values, therefore low amplitudes were excluded from the analysis. We constructed a correlation matrix, and used network analysis based on graph theory to obtain network parameters to further quantify the connectivity patterns and information flow in the brain. Wavelet transform

Using wavelet transform, wavelet spectra were constructed for each selected 2-second epoch of each subject. For both the healthy individuals and the epilepsy patients, the wavelet spectra were averaged over all 50 epochs. The wavelet spectra were compared between the healthy subjects and patients during discharge and nondischarge. Wavelet transform makes it possible to conduct spectrum analysis in a broad frequency range without losing time information [10]. The wavelet transform of a signal can be calculated by projecting the signal onto a basic wavelet (the mother wavelet) [29]. A Gaussian wavelet (Gabor mother wavelet) was used because it has the least spread in the time domain for a given spread in the frequency domain $[10,29]$. Time information is saved when conducting frequency analysis by making the mother wavelet expandable and contractable.

Wavelet-crosscorrelation analysis

We used wavelet-crosscorrelation analysis in order to calculate wavelet-crosscorrelation coefficients (WCC) between all 19 electrodes. It is a method that, while maintaining time information, makes it possible to investigate and visualize the correlation of neuronal activity between brain areas at various frequencies [10]. It therefore becomes possible to know how strong the functional connectivity is between the different areas in the brain for a wide range of frequencies. It is a combination of wavelet transform and crosscorrelation analysis, and displays how much the nature and shape of two signals resemble each other at different frequencies. WCC values range from 0 to 1 . The closer the WCC value is to 1 , the more the two signals are similar in terms of shape and nature; the closer the value is to 0 , the more different the two signals. Wavelet-crosscorrelation analysis is a powerful tool to analyze non-stationary data, for example EEGs that have sudden transient changes such as epileptiform discharges $[10,29]$. The wavelet-crosscorrelation function $W C_{x, y}(\alpha, \tau)$ is defined as [10]

$$
C_{x, y}(a, \tau)=\lim _{T \rightarrow \infty} \frac{1}{2 T} \int_{-T}^{T} \overline{W_{x}(b, a)} W_{y}(b+\tau, a) d b,
$$

where $T$ represents the period, $a$ the scaling parameter, $b$ the parameter that shifts the mother wavelet, and $\tau$ the time-lag in the wavelet space. $W C_{x, y}(\alpha, \tau)$ is complexvalued and consists of a real part $R W C_{x, y}(\alpha, \tau)$ and an imaginary part $I W C_{x, y}(\alpha, \tau) . R W C_{x, y}(\alpha, \tau)$ can be used to express the strength of the correlation between two signals $x$ and $y . \overline{W_{x}(b, a)}$ represents the complex conjugate. The wavelet-crosscorrelation coefficient $W R_{x y}(\alpha, \tau)$ from 
the real part of the wavelet-crosscorrelation function $R W C_{x, y}(\alpha, \tau)$ is [10]

$$
W R_{x, y}(a, \tau)=\frac{\left|R W C_{x, y}(a, \tau)\right|}{\sqrt{R W C_{x}(a, 0) R W C_{y}(a, 0)}} .
$$

The WCC were calculated from the numerical EEG data using an original program of Matlab R2017b. WCC values were analyzed in the alpha 1 bandwidth, averaged over $8.07 \mathrm{~Hz}$ to $10.87 \mathrm{~Hz}$. WCC values below 0.3 were not included in the analysis because low values may not be meaningful [29]. The WCC values were calculated between all 19 electrode channels (171 channel pair connections were possible). Each functional interaction between an electrode pair corresponds to $1 \mathrm{WCC}$ value. The calculated WCC values were constructed into a functional brain network by using a Tcl/TK program. In the constructed functional brain graphs, the vertices represent the electrode channels, and the edges correspond to the functional interactions (WCC values) between the electrodes. An interaction between 2 vertices was considered an edge only if the WCC value is above a certain threshold, otherwise no edge was shown. Choosing low threshold values would give rise to too many edges, resulting in difficult visual interpretation of each graph. A WCC threshold of 0.8 was therefore fixed over all graphs to reveal the strongest correlation strengths in each graph.

Statistical analysis of WCC

All statistical analyses were performed using SPSS Statistics 24. The $171 \mathrm{WCC}$ values were first averaged over all epochs. To directly compare the difference of the WCC over the entire functional network between healthy and patients, we used analysis of variance (ANOVA) with Bonferroni corrections on all $171 \mathrm{WCC}$ values. To investigate the individual differences between patients, the 171 WCC values were averaged over 5 epochs per patient before performing ANOVA.

\section{Graph theoretical analysis}

Graph theory was used to analyze the constructed brain networks. For each epoch, using an original program of Matlab R2017b, all calculated 171 WCC values were first converted to square 19x19 correlation matrices. The rows and columns of each correlation matrix represented the electrode channels, and the entries represented the WCC values. To compute the parameters of the network, we input the correlation matrices in the Matlab toolbox GRETNA. The correlation matrix was thresholded into a binary adjacency matrix by using the sparsity threshold method. An edge was present if the correlation value was above the threshold (value 1), otherwise an edge was absent (value 0). Sparsity is defined as the number of existing edges in the network divided by the maximum number of edges possible in the network [24,26,30]. We did not use the above mentioned WCC threshold of 0.8 , because setting a sparsity threshold ensures that all graphs of all subjects have the same number of edges and degree distribution. Therefore, alterations in the topology are solely reflected by differences in network measures between healthy subjects and patients [7,31].

We used a range of connectivity sparsity thresholds (10 to $40 \%$ ) with an increment of $1 \%$, and repeated the network analysis at each sparsity threshold value. This range of sparsity thresholds was chosen to avoid non-significant or spurious connections between vertices [26]. Low sparsity thresholds guarantee that edges with high correlation strengths (stronger, significant connections) remain, which may be more meaningful for our analysis [21,32].

Networks are characterized by several parameters that can quantify the network topology. Two of the most commonly calculated global measures are the clustering coefficient $C$ and the characteristic path length $L$. The local clustering coefficient $C_{i}$ of vertex $i$ with degree $k_{i}$ (the number of links departing from vertex $i$ ) is defined as the ratio of the number of existing edges $\left(e_{i}\right)$ between neighbor vertices of $i$, and the maximum possible number of edges between neighbor vertices of $i$ [14]. To obtain the mean $C$ of a graph with $N$ vertices, $C_{i}$ is averaged over all vertices. $C$ is defined as $[14,15,32]$

$$
C=\frac{1}{N} \sum_{i=1}^{N} C_{i}=\frac{1}{N} \sum_{i=1}^{N} \frac{2 e_{i}}{k_{i}\left(k_{i}-1\right)} .
$$

The clustering coefficient can be considered a measure of the segregation of the network, and indicates the local interconnectedness and cliquishness within the network [32]. To evaluate the properties of a real-life network, $C$ must be compared to the clustering coefficient estimated in a random network with the same number of vertices, edges, and degree distribution of the network of interest [33,34]. The normalized clustering coefficient $\gamma=C / C_{\text {rand }}$ is the ratio between the clustering coefficient $C$ of the actual network, and $C_{\text {rand }}$, the clustering coefficient of the corresponding surrogate random network that has the same number of vertices, edges and degree distribution [18, 33, 34].

The shortest path length or distance $d_{i, j}$ between two vertices $i$ and $j$ is the minimum number of edges that have to be traveled to go from $i$ to $j$ [14]. The characteristic path length $L$ is the mean of the path lengths between all possible pairs of vertices in the graph $[14,15,32]$ :

$$
L=\frac{1}{N(N-1)} \sum_{i, j \in N, i \neq j} d_{i, j} .
$$

The characteristic path length can be considered a measure of the integration of the network, and indicates how easy 
it is to transport information in the network [14,32]. The normalized characteristic path length $\lambda=L / L_{\text {rand }}$ is the ratio between the characteristic path length $L$ of the actual network, and $L_{\text {rand }}$, the characteristic path length of the corresponding surrogate random network $[18,33,34]$.

Regular networks are characterized by both a high clustering coefficient and a high characteristic path length, whereas random networks have both low $C$ and low $L[15,16]$. A small-world network has a high $C$ close to a regular network, but a very small $L$ close to that of a random network. In other words, small-world networks combine good local connectedness (segregation) with good global connectedness (integration) [16]. These properties of small-world networks present an attractive model for brain functional connectivity, as the brain supports both segregated/specialized information processing and integrated information processing [34]. Some studies have suggested to combine the above defined measures $\gamma$ and $\lambda$ of small-worldness into one measure. The index of small-worldness $\sigma$ is defined as

$$
\sigma=\frac{\gamma}{\lambda}
$$

As in small-world networks $\gamma>>1$ and $\lambda \geq 1$, a certain network is considered a small-world network if $\sigma>1$ $[18,34,35]$.

In order to compute $\gamma$ and $\lambda$, GRETNA is able to generate surrogate random networks with the same number of vertices, edges, and degree distributions as the actual networks. $\gamma, \lambda$, and $\sigma$ were calculated for each sparsity threshold between 10 and $40 \%$ with an increment of $1 \%$. In order to do statistical analysis, the calculated values of $\gamma, \lambda$, and $\sigma$ were averaged over all sparsity thresholds.

Vertices completely disconnected from the graph are an infinite number of paths separated from the other vertices, therefore in the computing of $\lambda$, the distance $d_{i, j}$ of disconnected vertices is infinite. For this reason, following previous studies, disconnected vertices were excluded while calculating $L$ and $\lambda$ in our analysis [36].

A common nodal measure is the nodal betweenness centrality $B_{i}$. The betweenness centrality $B_{i}$ of vertex $i$ is the ratio between all shortest paths between vertices $j$ and $k$ that run through $i$, and all shortest paths between $j$ and $k$ $\left(n_{j, k}\right)$ (equation 6) $[14,26,37] . B_{i}$ is an index of the relative importance of a vertex in the network, and also reflects the consequences of a loss of a certain vertex [14].

$$
B_{i}=\sum_{j, k \in N, j \neq k} \frac{n_{j, k}(i)}{n_{j, k}} .
$$

$B_{i}$ was calculated using GRETNA. To investigate the nodal characteristics of each vertex, a fixed sparsity threshold of
$30 \%$ was chosen because this threshold value ensures all vertices are included in the functional networks and the number of false positive paths are minimized, and may optimize interregional correlation strengths and therefore be biologically plausible [24,34,37]. An original Matlab program was used to visualize the $B_{i}$ values of all vertices in a brain graph. To define the hubs (vertices with high degree $[14,32])$ of the network, we computed the normalized betweenness centrality $b_{i}=B_{i} / B$ for each vertex [24,26,37]. $B$ is the average betweenness centrality of all the vertices of the network. $b_{i}$ is a measure that captures the influence of a vertex over information flow between other vertices in the network. Following previous studies, vertices with values of $b_{i}>1.5$ were defined as hubs [24,37].

Statistical analysis of the network parameters

The calculated network measures $\gamma, \lambda$, and $\sigma$ were first averaged over all sparsity threshold values. ANOVA with Bonferroni corrections was used on all 50 epochs to statistically compare the difference of the network parameters over the entire functional network between healthy and patients. For $30 \%$ sparsity threshold value, ANOVA with Bonferroni corrections was used to compare the electrodes with the highest $b_{i}$.

\section{RESULTS}

\subsection{EEGs}

Figure 2(a) shows the EEG of a healthy subject. Alpha waves mainly appeared in the occipital region of the brain (electrodes $\mathrm{O} 1$ and $\mathrm{O} 2$ ) during eyes closed (normal situation). Figures 2(b) and 2(c) show the interictal EEG of a patient at the time of epileptiform discharge, and during of non-discharge, respectively. Epileptiform discharges (theta bursts) sometimes prominently appeared in the interictal EEG of epilepsy patients over many channels.

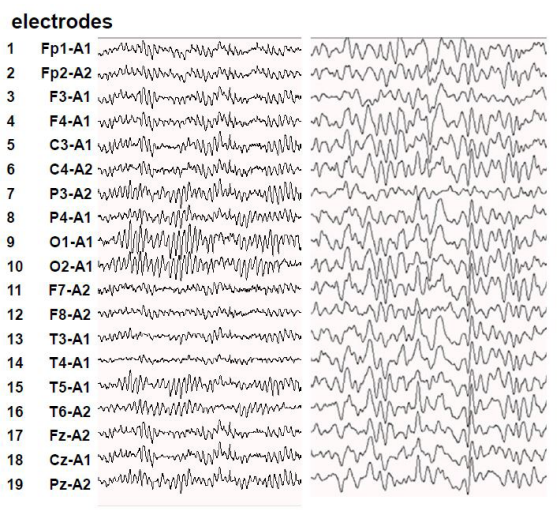

(a) healthy

(b) discharge

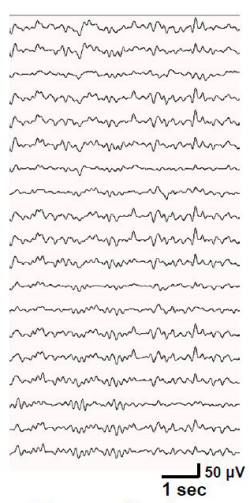

(c) non-discharge

Figure 2: EEG of an epoch of (a) a healthy individual; (b) a patient during epileptiform discharge; and (c) a patient during non-discharge. 


\subsection{Wavelet spectra}

Figure 3 shows the mean wavelet spectra of the mean of all 502 -second epochs of the healthy subjects (Figure 3(a)), the patients at the time of epileptiform discharge (Figure 3(b)), and at the time of non-epileptiform discharge (Figure 3(c)). The wavelet spectra are shown at all 19 electrodes according to the International 10-20 electrode positions. The abscissa shows the time in seconds, and the ordinate shows the frequency in $\mathrm{Hz}$. The color bar represents the lowest and the highest spectral value for all electrodes. In the healthy subject, high spectral activity only appeared in $\mathrm{O} 1$ and $\mathrm{O} 2$. In the patient during discharge, very high spectral activity widely appeared in many electrodes. At the time of non-discharge, high spectral activity appeared in some electrodes.

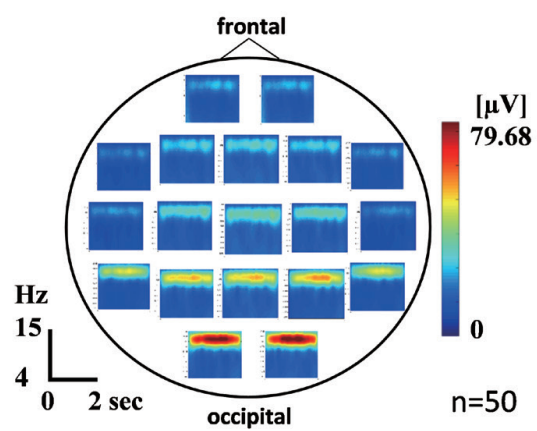

(a) Healthy subject

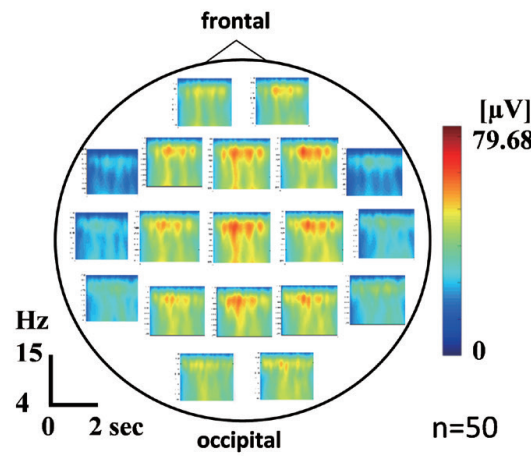

(b) Discharge

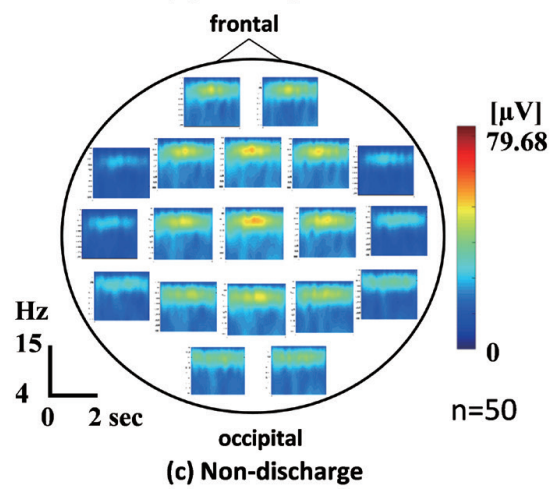

Figure 3: Mean wavelet spectra at all electrodes of a healthy individual (a), a patient at the time of discharge (b) and at the time of non-epileptiform discharge (c). The color bar on the right of the figure represents the lowest and the highest spectral value for all 19 electrodes.

\subsection{Wavelet-crosscorrelation analysis}

Figure 4 shows brain graphs that plot the mean WCC values of all 50 epochs of all healthy subjects (Figure 4(a)), and all patients during epileptiform discharge (Figure 4(b)), and at the time of non-discharge (Figure 4(c)) in the alpha 1 band. The WCC values of the healthy subjects were the highest (the correlation was the strongest) in the frontal and occipital areas of the brain. In the patients, during both discharge and non-discharge, the strongest connections were seen in the left central and left parietal areas of the brain. Figure 5 shows the mean WCC value between all 19 electrodes of all epochs of the healthy controls, and the mean WCC value between all 19

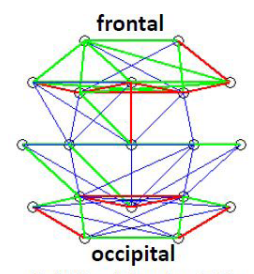

(a) Healthy $(n=50)$

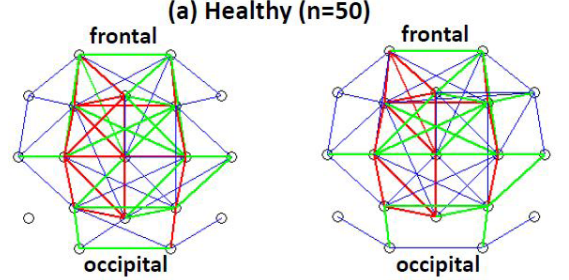

(b) Discharge $(n=50)$

(c) Non-discharge $(n=50)$

$0.80 \leqq W C C<0.85 ; \quad 0.85 \leqq W C C<0.90 ; \quad 0.90 \leqq W C C \leqq 1.00$

Figure 4: Brain graphs of the mean WCC values of all 50 epochs of all healthy subjects (a), of all patients at the time of discharge (b), and non-discharge (c).

Blue displays $0.80 \leq \mathrm{WCC}<0.85$, green displays $0.85 \leq \mathrm{WCC}<0.90$, and red displays $0.90 \leq \mathrm{WCC} \leq 1.00$.

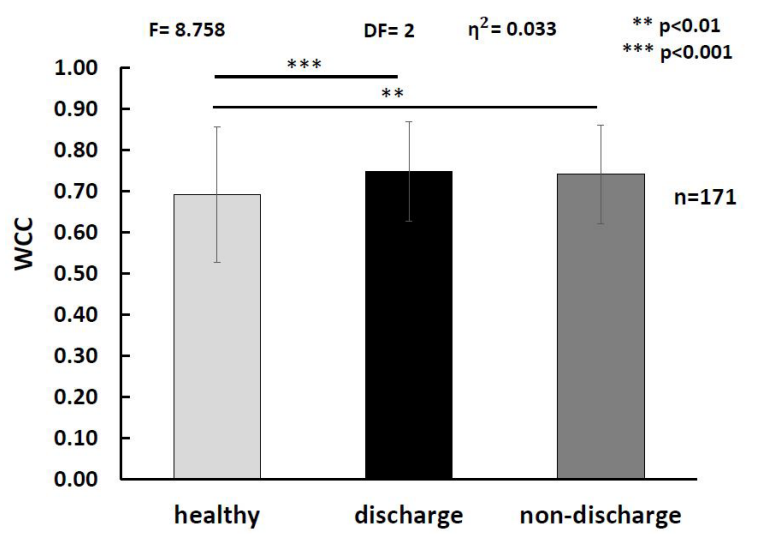

Figure 5: The mean WCC value between all 19 electrodes of all 50 epochs in the alpha 1 bandwidth for all healthy subjects, and all epilepsy patients during discharge and non-discharge.

The p-values, F-value, degrees of freedom (DF), and effect size $\left(\eta^{2}\right)$ are shown above the figure. The error bars represent the standard deviations of the mean WCC values. The wide standard deviation bars reflect high variation of the data ( $\mathrm{n}=171$ is the sample size). 
electrodes of all epochs of the epilepsy patients (during discharge and non-discharge) in the alpha 1 bandwidth. The mean WCC value in the patients at the time of both discharge $(p<0.001)$ and non-discharge $(p<0.01)$ was significantly higher than the mean WCC value in the brain of healthy individuals. Figure 6 shows the WCC values averaged over 5 epochs for each individual patient.

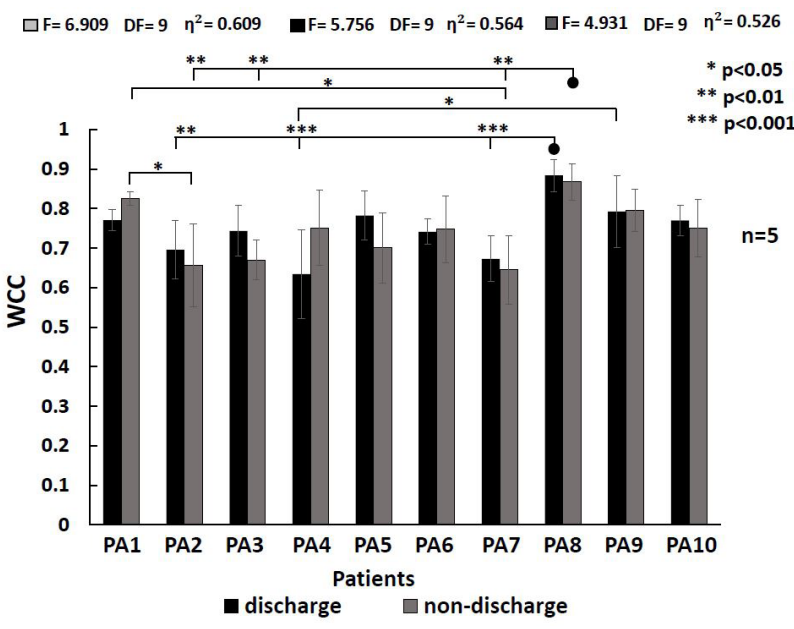

Figure 6: WCC values for each individual patient.

The error bars represent the standard deviations of the mean WCC values.
During both discharge and non-discharge, individual differences were visible.

\subsection{Graph theoretical analysis}

The global network measures are displayed in Figure 7. Figure 7(a) shows the comparison of the mean normalized clustering coefficient $\gamma$ of all 50 epochs (averaged over all sparsity thresholds) among the healthy subjects, the patients during discharge and during non-discharge. Significantly lower mean $\gamma$ values were seen in the patients during both discharge and non-discharge compared to the healthy subjects $(\mathrm{p}<0.001)$. Figure $7(\mathrm{~b})$ shows the mean normalized characteristic path length $\lambda$ of all epochs for all thresholds. The mean $\lambda$ values of the brain network of the patients at the time of both discharge and non-discharge $(\mathrm{p}<0.001)$ were significantly lower than the mean $\lambda$ value of the brain network of the healthy subjects. Figure 7(c) shows the comparison of the mean small-worldness $\sigma$ of all epochs and thresholds. Significantly lower mean $\sigma$ values were observed in the brain network of the patients during both discharge and non-discharge than in the healthy subjects $(\mathrm{p}<0.01)$. Figures 8(a), 8(b), and 8(c) show the change of network indices $\gamma, \lambda$, and $\sigma$, respectively in function of increasing

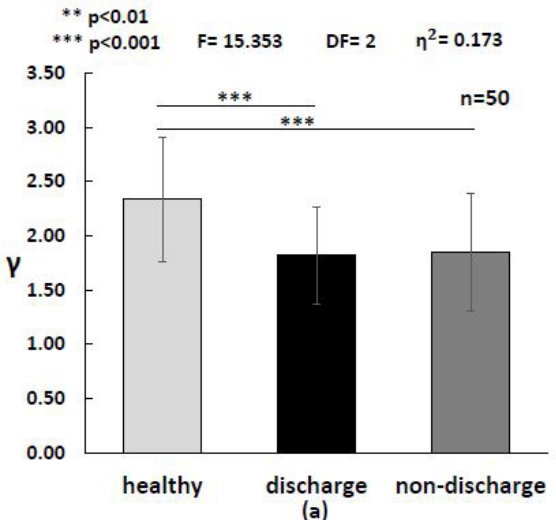

(a)

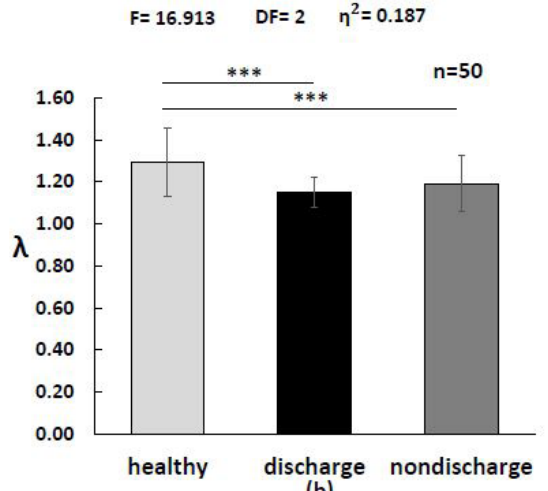

(b)

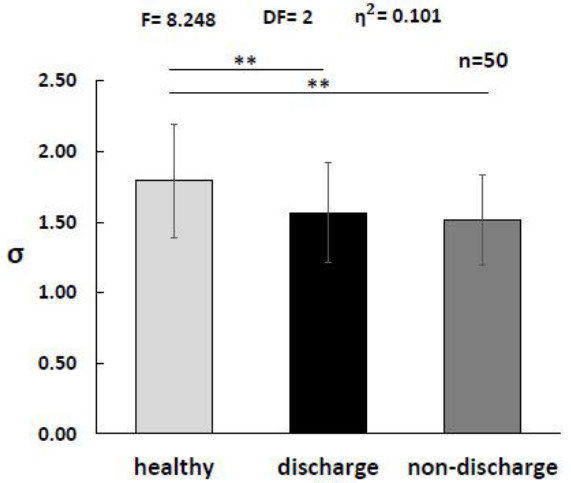

(c)

Figure 7: The mean values of the network measures $\gamma(\mathrm{a}), \lambda(\mathrm{b})$, and $\sigma(\mathrm{c})$ of all 50 epochs for the healthy subjects, and the patients during discharge and non-discharge.

All 3 parameters were averaged over all sparsity thresholds (10\% to 40\%). The error bars represent the standard deviations of the mean $\gamma(\mathrm{a}), \lambda(\mathrm{b})$, and $\sigma$ (c) values. Each measure showed significantly lower values in the patient group during both discharge and non-discharge compared to the healthy individuals.

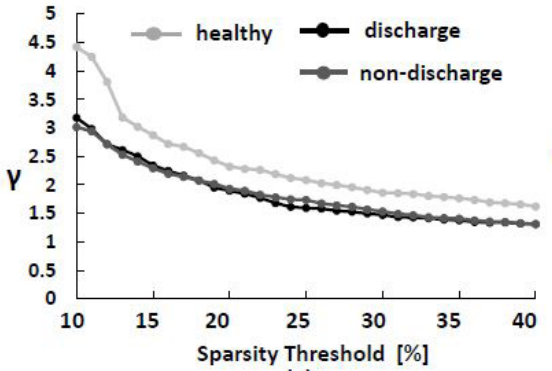

(a)

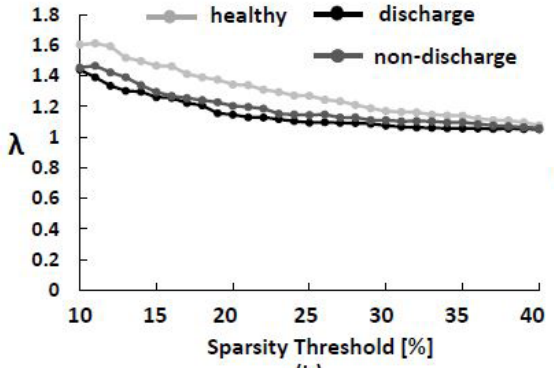

(b)

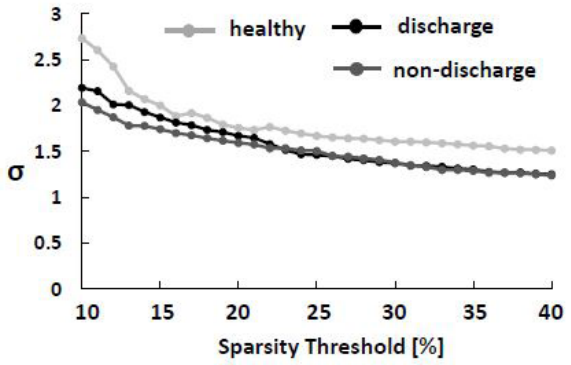

(c)

Figure 8: The change of network indices $\gamma(\mathrm{a}), \lambda(\mathrm{b})$, and $\sigma(\mathrm{c})$, in function of increasing threshold. 


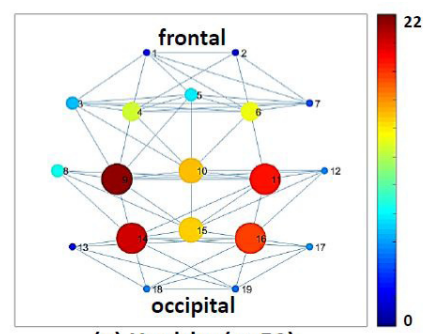

(a) Healthy $(n=50)$

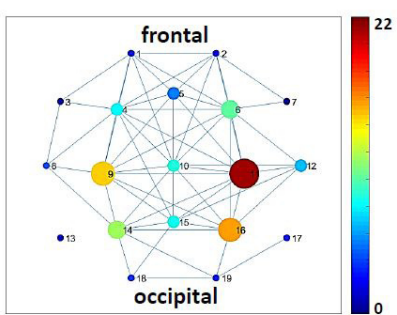

(b) Discharge $(n=50)$

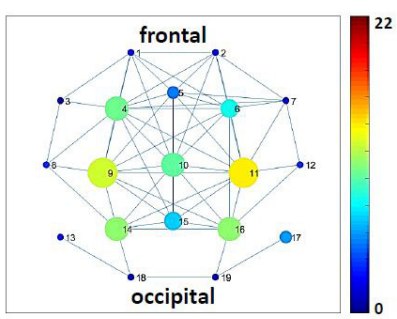

(c) Non-discharge $(n=50)$
Figure 9: Nodal betweenness centrality $B_{i}$ of all 19 vertices represented in a brain graph in the alpha 1 bandwidth of all healthy subjects (a), of all patients at the time of discharge (b), and non-discharge (c).

The color bar on the right shows the value of $B_{i}$.

sparsity threshold. All three measures are shown to decrease with increasing threshold in both healthy subjects and patients.

Figure 9 shows the distribution of the nodal betweenness centrality $B_{i}$ of all healthy subjects (Figure 9(a)), of all patients during epileptiform discharge (Figure 9(b)), and of all patients during non-discharge (figure 9(c)) in the alpha 1 bandwidth. The color bar on the right shows the values of $B_{i}$. In addition, the size of the nodes is proportional to the size of $B_{i}$. In the healthy subjects and the patients during non-discharge, high centralities were found in the left, middle, and right central and parietal areas. On the other hand, during discharge, high centrality was observed mainly in the right central area, and there was no high centrality in the middle area.

Figure 10 shows the normalized betweenness centrality $b_{i}$ of the hub vertices $\left(b_{i}>1.5\right)$ for the healthy subjects and the patients during discharge and non-discharge. F3 was a hub region in the brain network of the patients only during non-discharge, and had a significantly higher $b_{i}$ than during discharge $(\mathrm{p}<0.05)$. C4 was a hub region for all subjects, but a significantly higher $b_{i}$ was observed during discharge compared to non-discharge $(\mathrm{p}<0.05)$ and healthy $(\mathrm{p}<0.001)$. In the patients' brain network during discharge, $\mathrm{Cz}$ was not a hub region, as opposed to during non-discharge and the control group. $\mathrm{Pz}$ was only a hub area in the healthy subjects.

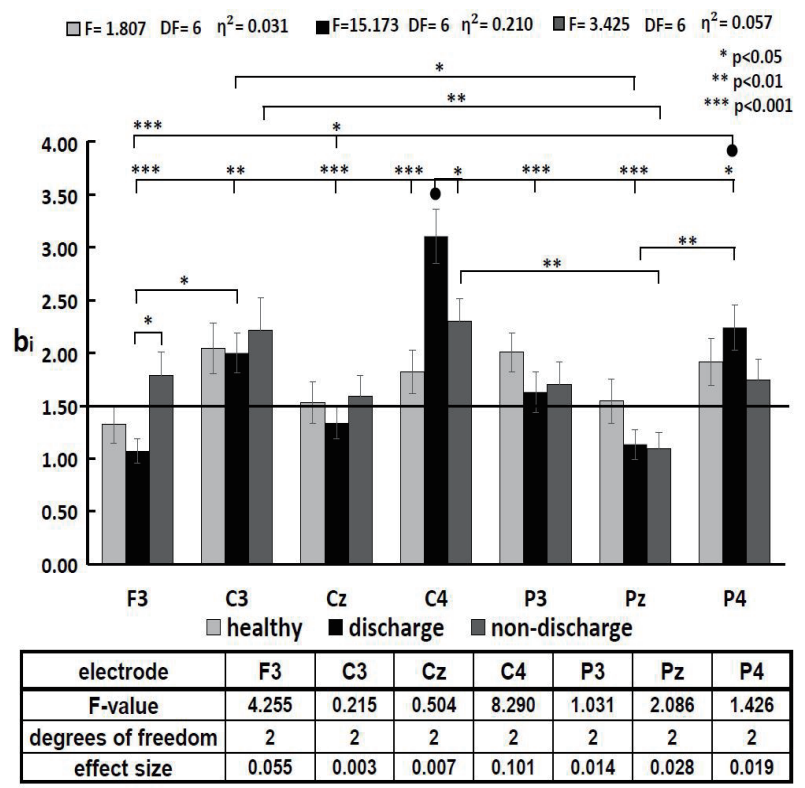

Figure 10: Normalized betweenness centrality $b_{i}$ of the vertices displaying the highest values. Vertices with $b_{i}$ values $>1.5$ were considered hubs of the network.

The error bars represent the standard deviations of the mean $b_{i}$ values.

\section{DISCUSSION}

In this study, we analyzed scalp recorded EEG data by using wavelet-crosscorrelation analysis to derive networks of functional connectivity, and applied graph theory to analyze the spatial distribution of the functional networks. We compared the interictal functional network of the brain of epilepsy patients during epileptiform discharge with during non-discharge. Our main findings were that the functional connectivity strength, as well as the functional network configuration in the brain of the epilepsy patients, both at the time of discharge and at the time of non-epileptiform discharge, were altered compared to the healthy subjects. The novel finding of this study is that even though in the patients, the functional connectivity and small-worldness of the brain did not show significant differences between discharge and non-discharge, the centrality and the spatial distribution of the hub regions were different between during epileptiform discharge and during non-discharge.

After performing wavelet transform on the EEG data, the wavelet spectra showed large differences between the healthy subjects and the patients. The spectral activity at the time of discharge is much higher, and is spread over the theta band in addition to the alpha band compared to during non-discharge and in the healthy brain. Visualizing EEG in a map of wavelet spectra shows at which frequencies the highest spectral activity occurs, and could be helpful for 
clinical doctors to more easily interpret the neuronal activity between different brain regions [29].

Wavelet-crosscorrelation analysis allowed us to construct functional connectivity networks, and reveal the strongest correlation strengths for each group. Visualizing functional connectivity patterns might reveal a lot about brain dynamics and brain function. Functional networks may reveal connections or information beyond the anatomical structure [14]. It has been reported that functional connections undergo spontaneous fluctuations on very short timescales, reflecting responses to sensory input and cognitive tasks $[8,38]$. On longer timescales however, functional connectivity networks have been found to be robust [39]. In our results, strong connections seemed to be clustered in the frontal and occipital areas in the healthy subjects, whereas in the patients, strong connections appeared widely over the entire network. This finding is similar to our previous study, which suggested that the communication in the brain of neuropsychiatric patients may be only possible if all regions of the brain are actively involved [29]. Similar to our previous study, the brain of the epilepsy patients showed significantly higher total functional connectivity compared to the healthy subjects.

Previous research based on EEG has also found increased connectivity in the interictal functional networks in epilepsy patients [2,6,40]. An increased functional connectivity has been reported in the temporal lobes in individuals with temporal lobe epilepsy compared to healthy subjects [20]. Since cognitive dysfunction has been reported in epilepsy patients [21], it could be the case that the higher connectivity in the brain of epilepsy patients might be a compensation mechanism for the loss of optimal brain function. Our study showed a small, non-significant increase in functional connectivity (WCC) in the alpha 1 bandwidth during epileptiform discharge compared to non-discharge in the patients, which is similar to a study of Yamaguchi et. al. based on EEG recordings in epilepsy patients [41]. By using graph theoretical analysis, our study examined whether or not in the alpha bandwidth, the brain network topology of epilepsy patients has significant differences between discharge and non-discharge.

Our study examined the small-worldness of the brain network of the healthy subjects, and the patients during epileptiform discharge and during non-discharge. It is believed that the human brain functional network develops for an optimal balance between segregation (specialized processing within dense interconnected brain areas) and functional integration (the ability to rapidly combine specialized information from brain regions) between these specialized brain areas $[9,14,32]$. This optimal balance has been referred to as complexity $[13,42]$. The clustering coefficient of a network can be seen as a measure of functional segregation, whereas the characteristic path length can be considered a measure of functional integration [32]. Brain networks with a small-world topology are considered to be optimal for brain functioning and maximizing complexity $[8,16,43]$. Previous studies have suggested a loss of optimal brain function due to a loss of the optimal small-world topology in the brain network of individuals with neuropsychiatric diseases [7, 19].

Our results showed that the network topology of the healthy subjects had a small world configuration $(\gamma>>1$, $\lambda \geq 1$, and $\sigma>1$ ). Networks can gradually transition from a completely small-world topology to a completely random topology $(\gamma \geq 1, \lambda \approx 1$, and $\sigma \geq 1)[15,16]$. Our findings showed a loss of small-world topology toward a more random, less optimal configuration (decreased clustering and an even shorter path length) in the brain network of the epilepsy patients. These results suggest that the brain network of the epilepsy patients is less optimal and might function less efficient. Decreased $\gamma, \lambda$, and $\sigma$ in the interictal period of epilepsy patients were also observed in fMRI and electrocorticography studies [20,44]. A study based on MEG concluded that a very low path length may be responsible for a tendency to excessive synchronization and epileptic seizures in brain tumor patients [11]. Our results are in line with these previous network studies. Synchronization of neurons in healthy brain networks is important for normal functioning and information processing [9]. However, a change in topology due to brain disease might further lower the brain's threshold for synchronization [9], which increases susceptibility to seizures. It has been suggested that brain networks are located dynamically on a critical point between local neuronal synchronization to global synchronization [45]. Several model studies support the notion that a more random topology causes a network to synchronize more easily due to lowering the threshold for synchronization [46-48]. The cause of the randomization of the network could be due to cell loss inflicted by epileptic lesions, resulting in fewer connections [43]. Interestingly, a more random network topology was also found at the time of non-discharge. This suggests a continuous brain dysfunction of epilepsy patients, and continuous susceptibility for seizures during the interictal period.

In this study, a decreased nodal centrality of some regions in the patients' brain network during epileptiform discharge was revealed. The brain network during nondischarge showed a hub distribution closer to that of the 
controls. Hub areas are important to facilitate information transfer in the brain network $[8,14]$, making hubs points of vulnerability to brain dysfunction $[37,49]$. A change of spatial distribution of hubs may result in a less efficient information flow in the brain network, therefore disrupting the functional connectivity of the brain $[33,49]$. The brain graphs displaying the nodal centrality in the patients during discharge showed a loss of hubs mainly along the z-line. This may suggest a separate, less efficient flow of information in the left and right hemispheres than in healthy subjects. On the other hand, a very high centrality was found in the right hemisphere (C4) during discharge. This may be interpreted as a compensation mechanism for the inefficient information transfer.

Several measures were computed in this study. The small-worldness $\sigma$ is a powerful index because it combines the indices $\gamma$ and $\lambda$, and tells more about the topological organization and function of the brain than correlation indices such as WCC. While the optimal network topology of the brain has been reported as being small-world, the optimal functional connectivity in the brain is not clearly defined $[8,16]$. This study showed lower small-worldness in the patients' brains even at the time of non-discharge activity, therefore the index $\sigma$ might aid to distinguish the healthy brain from the epileptic brain.

In order to diagnose epilepsy with higher sensitivity, it might be meaningful to develop a computer-aided diagnosis system to automatically distinguish interictal epileptiform discharge epochs from non-discharge epochs using for example machine learning techniques or automated detection algorithms. The difference in the centrality index between discharge and non-discharge epochs found in our study might be used in future machine learning algorithms.

In conclusion, our results suggest that graph theoretical analysis of the human brain functional connectivity network derived from the correlation between EEG time series might provide a deeper understanding of the pathophysiological mechanisms underlying brain dysfunction in neuropsychiatric disorders such as epilepsy. The present study provides further evidence for a loss of the optimal small-world configuration of the functional network of epilepsy patients in the direction of a more random network. This more random network seems to push the dynamics of the brain from an optimal intellectual function toward a pathological, seizure-prone state. Furthermore, we have shown that this disturbance in the functional connectivity may be persistently present in the interictal period even when no epileptiform discharges are visible in the EEG. Network indices might therefore aid to distinguish the epileptic brain from the healthy brain, and aid to diagnose epilepsy with a higher sensitivity. The nodal betweenness centrality index might aid to distinguish EEGs at the time of epileptiform discharges from EEGs at the time of non-discharges.

\section{ACKNOWLEDGEMENTS}

This work was supported by JSPS KAKENHI Grant number JP16K01367.

\section{REFERENCES}

1. Vecchio, F., Miraglia, F., and Rossini, P.M.; Connectome: Graph theory application in functional brain network architecture, Clinical Neurophysiology Practice, 2, pp.206-213, 2017.

2. Douw, L., et al.; Quantitative EEG as a predictive biomarker for Parkinson disease dementia, Neurology, 77(2), pp.118-124, 2011.

3. Smith, S.M. J.; EEG in the diagnosis, classification, and management of patients with epilepsy, Journal of Neurology, Neurosurgery \& Psychiatry, 76(II), pp.ii2-ii7, 2005.

4. Pillai, J., and Sperling, M.R.; Interictal EEG and the diagnosis of epilepsy, Epilepsia, 47(1), pp.14-22, 2006.

5. Faust, O., et al.; Wavelet-based EEG processing for computer-aided seizure detection and epilepsy diagnosis, Seizure, 26, pp.56-64, 2015.

6. van Diessen, E., et al.; Functional and structural brain networks in epilepsy: What have we learned?, Epilepsia, 54(11), pp.1855-1865, 2013.

7. Stam, C.J., et al.; Small-world networks and functional connectivity in Alzheimer's disease, Cerebral Cortex, 17(1), pp.92-99, 2007.

8. Bullmore, E., and Sporns, O.; Complex brain networks: Graph theoretical analysis of structural and functional systems, Nature Reviews Neuroscience, 10(3), pp.186-198, 2009.

9. Reijneveld, J.C, et al.; The application of graph theoretical analysis to complex networks in the brain, Clinical Neurophysiology, 118(11), pp.2317-2331, 2007.

10. Mizuno-Matsumoto, Y., et al.; Wavelet-crosscorrelation analysis: Non-stationary analysis of neurophysiological signals, Brain Topography, 17(4), pp.237-252, 2005.

11. Bartholomei, F., et al.; Disturbed functional connectivity in brain tumour patients: Evaluation by graph analysis of synchronization matrices, Clinical Neurophysiology, 117(9), pp.2039-2049, 2006. 
12. Fair, D.A., et al.; Development of distinct control networks through segregation and integration, PNAS, 104(33), pp.13507-13512, 2007.

13. He, Y., Chen, Z.J., and Evans, A.C.; Small-world Anatomical networks in the human brain revealed by cortical thickness from MRI, Cerebral Cortex, 17(10), pp.2407-2419, 2007.

14. Stam, C.J., and Reijneveld, J.C.; Graph theoretical analysis of complex networks in the brain, Nonlinear Biomedical Physics, 1, 3, 2007.

15. Barabasi, A.L., and Posfai, M.; Network science; 4th edition, Cambridge University Press, Cambridge, United Kingdom, 2016.

16. Watts, D.J., and Strogatz, S.H.; Collective dynamics of 'small-world' networks, Nature, 393(4), pp.440-442, 1998.

17. Strogatz, S.H.; Exploring complex networks, Nature, 410(8), pp.268-276, 2001.

18. Achard, S., et al.; A resilient, low-frequency, small-world human brain functional network with highly connected association cortical hubs, Journal of Neuroscience, 26(1), pp.63-72, 2006.

19. Micheloyannis, S., et al.; Small-world networks and disturbed functional connectivity in schizophrenia, Schizophrenia Research, 87(1-3), pp.60-66, 2006.

20. Liao, W., et al.; Altered functional connectivity and small-world in mesial temporal lobe epilepsy, PLoS ONE, 5(1), e8525, 2010.

21. Vlooswijk, M.C. G., et al.; Loss of network efficiency associated with cognitive decline in chronic epilepsy, Neurology, 77(10), pp.938-944, 2011.

22. Kramer, M.A., Kolaczyk, E.D., and Kirsch, H.E.; Emergent network topology at seizure onset in humans, Epilepsy Research, 79(2-3), pp.173-186, 2008.

23. Japan Society of Kansei Engineering, https://www. jske.org/abouts [11/13]. (in Japanese)

24. He, Y., Chen. Z., and Evans, A.; Structural insights into aberrant topological patterns of large-scale cortical networks in Alzheimer's disease, Journal of Neuroscience, 28(18), pp.4756-4766, 2008.

25. Boersma, M., et al.; Network analysis of resting state EEG in the developing young brain: Structure comes with maturation, Human Brain Mapping, 32(3), pp.413-425, 2011

26. Liu, Z., et al.; Changes in Topological organization of functional PET brain network with normal aging, PLoS ONE, 9(2), e88690, 2014.

27. Vecchio, F., et al.; Human brain networks in physiological aging: A graph theoretical analysis of cortical connectivity from EEG data, Journal of Alzheimer's
Disease, 41(4), pp.1239-1249, 2014.

28. Vecchio, F., et al.; Cortical connectivity in frontotemporal focal epilepsy from EEG analysis: A study via graph theory, Clinical Neurophysiology, 126(6), pp.1108-1116, 2015.

29. Carpels, S. M.A., et al.; Comparing the features of the diffuse alpha pattern with the normal alpha pattern using wavelet-crosscorrelation analysis, International Journal of Affective Engineering, 19(1), pp.21-30, 2020.

30. Wang, J., et al.; GRETNA: A graph theoretical network analysis toolbox for imaging connectomics, Frontiers in Human Neuroscience, 9, 386, pp.1-16, 2015.

31. Achard, S., and Bullmore, E.; Efficiency and cost of economical brain functional networks, PLoS ONE, 3(2), e17, 2007.

32. Rubinov, M., and Sporns, O.; Complex network measures of brain connectivity: Uses and interpretations, Neuroimage, 52(3), pp.1059-1069, 2010.

33. Sporns, O., and Zwi, J.D.; The small world of the cerebral cortex, Neuroinformatics, 2(2), pp.145-162, 2004.

34. Bassett, D. S., and Bullmore, E.; Small-world brain networks, Neuroscientist, 12(6), pp.512-523, 2006.

35. Humphries, M.D., and Gurney, K.; Network 'smallworld-ness': A quantitative method for determining canonical network equivalence, PLoS ONE, 3(4), e0002051, 2008.

36. Supekar, K., et al.; Network analysis of intrinsic functional brain connectivity in Alzheimer's disease, PloS ONE, 4(6), e1000100, 2008.

37. Qiu, X., et al.; Positron emission tomography reveals abnormal topological organization in functional brain network in diabetic patients, Frontiers in Neuroscience, 10, 235, pp.1-11, 2016.

38. Honey, C.J., et al.; Network structure of cerebral cortex shapes functional connectivity on multiple time scales, PNAS, 104(24), pp.10240-10245, 2007.

39. Kramer, M.A., et al.; Emergence of persistent networks in long-term intracranial EEG recordings, Journal of Neuroscience, 31(44), pp.15757-15767, 2011.

40. Stam, C. J., and van Straaten, E. C.W.; The organization of physiological brain networks, Clinical Neurophysiology, 123(6), pp.1067-1087, 2012.

41. Yamaguchi, K., et al.; The correlation between the sites of the brain for different bandwidths of EEG in interictal paroxysmal rhythmic activity: Using wavelet-crosscorrelation analysis, Transactions of Japan Society of Kansei Engineering, 16(2), pp.245-252, 2017. (in Japanese) 
42. Tononi, G., Sporns, O., and Edelman, G. M.; A measure for brain complexity: Relating functional segregation and integration in the nervous system, PNAS, 91(11), pp.5033-5037, 1994.

43. van Dellen, E., et al.; Long-term effects of temporal lobe epilepsy on local neural networks: A graph theoretical analysis of corticography recordings, PLoS ONE, 4(11), e8081, 2009.

44. Ponten, S., Bartolomei, F., and Stam, C. J.; Small-world networks and epilepsy: Graph theoretical analysis of intracerebrally recorded mesial temporal lobe seizures, Clinical Neurophysiology, 118(4), pp.918-927, 2007.

45. Bassett, D.S., et al.; Adaptive reconfiguration of fractal small-world human brain functional networks, PNAS, 103(51), pp.19518-19523, 2006.

46. Chavez, M. et al.; Synchronizing weighted complex networks, Chaos, 16(1), 015106, 2006.

47. Percha, B., Dzakpasu, R., and Zochowski, M.; Transition from local to global phase synchrony in small world neural network and its possible implications for epilepsy, Physical Review, 72(3), 031909, 2005.

48. Netoff, T. I., et al.; Epilepsy in small-world networks, Journal of Neuroscience, 24(37), pp.8075-8083, 2004.

49. Sporns, O., Honey, C. J., and Kotter, R.; Identification and classification of hubs in brain networks, PLoS ONE, 2(10), e1049, 2007.

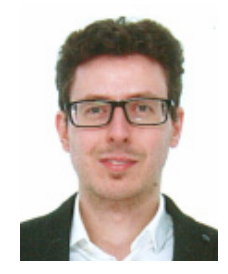

Steven M.A. CARPELS (Member)

Steven M.A. Carpels received the B.S. and M.S. degrees in Science (Biology) from Ghent University in 2006 and 2008, respectively. He received the M.S. degree in Applied Informatics from the University of Hyogo in 2017. From 2017, he has been a student in the Ph.D. course of the Graduate School of Applied Informatics, University of Hyogo.

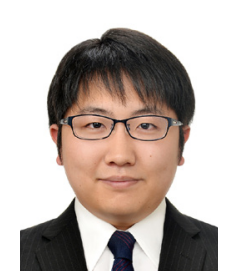

Yusuke YAMAMOTO (Non-member)

Yusuke Yamamoto received the B.S. degree in Medical Engineering and the C.E. degree from the Faculty of Biology-Oriented Science and Technology, Kindai University in 2018. He received the M.S. degree in 2020 and has been a student in the Doctoral course of the Graduate School of Applied Informatics, University of Hyogo.

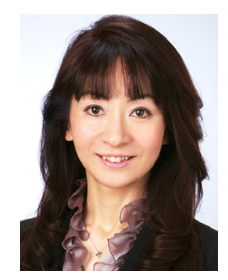

Yuko MIZUNO-MATSUMOTO (Member)

Yuko Mizuno-Matsumoto received the M.D. degree from Shiga University of Medical Science, Japan in 1991 and received Ph.D. degrees in medicine and engineering from Osaka University in 1996 and 2003, respectively. From 1999 to 2000, she was a Post-Doctoral Research Fellow in the Department of Neurology, Johns Hopkins University. From 2004 to 2011, she was an Associate Professor, and since 2011 a Professor in the Graduate School of Applied Informatics, University of Hyogo. Since 2019, she is a Professor in Osaka University. She is a Certified Psychiatrist of the Japanese Society of Psychiatry and Neurology, and a Certified Physician and Representative of the Japanese Society of Clinical Neurophysiology. 